\title{
Classification of general $n$-qubit states under stochastic local operations and classical communication in terms of the rank of coefficient matrix
}

\author{
Xiangrong $\mathrm{Li}^{1}$, Dafa $\mathrm{Li}^{2,3}$ \\ ${ }^{1}$ Department of Mathematics, University of California Irvine, Irvine, California 92697, USA \\ ${ }^{2}$ Department of Mathematical Sciences, Tsinghua University, Beijing, 100084, China \\ ${ }^{3}$ Center for Quantum Information Science and Technology, Tsinghua National Laboratory for Information \\ Science and Technology (TNList), Beijing, 100084, China
}

\begin{abstract}
We solve the entanglement classification under stochastic local operations and classical communication (SLOCC) for general $n$-qubit states. For two arbitrary pure $n$-qubit states connected via local operations, we establish an equation between the two coefficient matrices associated with the states. The rank of the coefficient matrix is preserved under SLOCC and gives rise to a simple way of partitioning all the pure states of $n$ qubits into different families of entanglement classes, as exemplified here. When applied to the symmetric states, this approach reveals that all the Dicke states $|\ell, n\rangle$ with $\ell=1, \ldots,[n / 2]$ are inequivalent under SLOCC.
\end{abstract}

\section{INTRODUCTION}

Entanglement, a key feature that distinguishes quantum information from classical information, has applications in cryptography, teleportation and quantum computation [1]. While the bipartite entanglement is well understood, the task of classifying multipartite entanglement beyond two qubits becomes increasingly difficult. To classify entangled states, some equivalence relation has to be introduced. Of particular importance is the equivalence under stochastic local operations and classical communication (SLOCC) since two states belonging to the same equivalence class can perform the same tasks of quantum information theory.

For three qubits, in terms of the local ranks of the reduced density matrices, it has been shown that there are six inequivalent SLOCC classes [2]. For four or more qubits, there exists an infinite number of inequivalent SLOCC classes. It is highly desirable to partition the infinite SLOCC classes into a finite number of families such that states belonging to the same family possess similar properties, according to some criteria for determining which family a given state belongs to. Considerable efforts have been undertaken over the last decade for the SLOCC entanglement classification of four-qubit states resulting in a finite number of families [3-6] or classes [7-12]. For more than four qubits, a few attempts have been made for SLOCC classification for subsets of the general $n$-qubit states such as the Greenberger-HorneZeilinger (GHZ)-type, W-type, and GHZ-W-type $n$-qubit states [13], symmetric $n$-qubit states [14-16], even $n$ qubit states [17, 18], and odd $n$-qubit states [19]. Despite these efforts, a SLOCC classification for general $n$-qubit states is still beyond reach.

Our aim is to solve the problem of SLOCC classification for all multipartite pure states in the general $n$-qubit case. To this end, we demonstrate that the rank of the coefficient matrix of a pure $n$-qubit state is invariant under SLOCC. SLOCC invariants for subsets of $n$-qubit states have been the subject of several recent studies [17 21]. In [17, 18], the invariant element is the determinant of coefficient matrices of even $n$ qubits. In [19], the invariant element is the rank of square matrices of size two constructed using three functions defined on the space of odd $n$ qubits.

We construct the coefficient matrices for general $n$ qubit states by arranging the coefficients in lexicographical order. For two states connected via local operations, their coefficient matrices are related through an equation. In the case where the local operations are invertible, the two states are said to be SLOCC equivalent and the two coefficient matrices have the same rank; i.e., the rank is preserved under SLOCC. The rank gives rise to a simple way of partitioning all the $n$-qubit states into different SLOCC families. For $n$-qubit symmetric Dicke states $|\ell, n\rangle$ with $\ell(\ell=1, \ldots,[n / 2])$ excitations, we show that the rank of the coefficient matrix of $|\ell, n\rangle$ is equal to $\ell+1$ and, therefore, all these states are inequivalent under SLOCC. Finally, composing the rank and permutations of qubits allows us to define subfamilies by cutting each family in pieces.

\section{SLOCC MATRIX EQUATION AND THE INVARIANCE OF THE RANK}

We write the state $\left|\psi^{\prime}\right\rangle$ of $n$ qubits as $\left|\psi^{\prime}\right\rangle=$ $\sum_{i=0}^{2^{n}-1} a_{i}|i\rangle$, where $|i\rangle$ are basis states and $a_{i}$ are coefficients. We associate to an $n$-qubit state $\left|\psi^{\prime}\right\rangle$ a $2^{[n / 2]} \times 2^{[(n+1) / 2]}$ coefficient matrix $M\left(\left|\psi^{\prime}\right\rangle\right)$ whose entries are the coefficients $a_{0}, a_{1}, \ldots, a_{2^{n}-1}$ arranged in ascending lexicographical order. To illustrate, we list below $M\left(\left|\psi^{\prime}\right\rangle\right)$ for $n=3$ :

$$
M\left(\left|\psi^{\prime}\right\rangle\right)=\left(\begin{array}{cccc}
a_{0} & a_{1} & a_{2} & a_{3} \\
a_{4} & a_{5} & a_{6} & a_{7}
\end{array}\right),
$$


and for $n=4$ :

$$
M\left(\left|\psi^{\prime}\right\rangle\right)=\left(\begin{array}{cccc}
a_{0} & a_{1} & a_{2} & a_{3} \\
a_{4} & a_{5} & a_{6} & a_{7} \\
a_{8} & a_{9} & a_{10} & a_{11} \\
a_{12} & a_{13} & a_{14} & a_{15}
\end{array}\right)
$$

We refer to the rank of the coefficient matrix $M\left(\left|\psi^{\prime}\right\rangle\right)$ as the rank of the state $\left|\psi^{\prime}\right\rangle$, denoted as $\operatorname{rank}\left(\left|\psi^{\prime}\right\rangle\right)$. We exemplify with the $n$-qubit $|\mathrm{GHZ}\rangle$ state $\frac{1}{\sqrt{2}}\left(|0\rangle^{\otimes n}+\right.$ $\left.|1\rangle^{\otimes n}\right)$, and we find $\operatorname{rank}(|\mathrm{GHZ}\rangle)=2$. It is clear that the rank of any $n$-qubit state ranges over the values $1,2, \ldots$, $2^{[n / 2]}$.

Theorem. Let $|\psi\rangle$ be another state of $n$ qubits with $|\psi\rangle=\sum_{i=0}^{2^{n}-1} b_{i}|i\rangle$ and $M(|\psi\rangle)$ be the corresponding coefficient matrix constructed in the same manner as was done for $M\left(\left|\psi^{\prime}\right\rangle\right)$. If the states $|\psi\rangle$ and $\left|\psi^{\prime}\right\rangle$ are related by

$$
\left|\psi^{\prime}\right\rangle=\mathcal{A}_{1} \otimes \mathcal{A}_{2} \otimes \ldots \otimes \mathcal{A}_{n}|\psi\rangle
$$

where the local operators $\mathcal{A}_{1}, \mathcal{A}_{2}, \ldots$, and $\mathcal{A}_{n}$ are not necessarily invertible, then the following matrix equation holds for general $n$ qubits:

$$
M\left(\left|\psi^{\prime}\right\rangle\right)=\left(\mathcal{A}_{1} \otimes \ldots \otimes \mathcal{A}_{[n / 2]}\right) M(|\psi\rangle)\left(\mathcal{A}_{[n / 2]+1} \otimes \ldots \otimes \mathcal{A}_{n}\right)^{T} .
$$

Equation (44) holds particularly true for two SLOCC equivalent states $|\psi\rangle$ and $\left|\psi^{\prime}\right\rangle$ which satisfy Eq. (3) along with the local operators $\mathcal{A}_{1}, \mathcal{A}_{2}, \ldots$, and $\mathcal{A}_{n}$ being invertible [2]. It follows from Eq. (44) that two SLOCC equivalent states have the same rank, in other words, the rank is invariant under SLOCC, thereby revealing that the rank is an inherent physical property. Therefore, if two states differ in their ranks, then they belong necessarily to different SLOCC equivalent classes. It should be noted that the converse does not hold; i.e., two states with the same rank are not necessarily equivalent.

We have the following two simple results: (i) The rank of a full separable state is always 1. (ii) The rank of a genuinely entangled state is always greater than 1 .

Remark 1. Taking the determinants of both sides of Eq. (44) for even $n$ yields [17, 18]:

$$
\operatorname{det} M\left(\left|\psi^{\prime}\right\rangle\right)=\operatorname{det} M(|\psi\rangle)\left[\operatorname{det}\left(\mathcal{A}_{1}\right) \ldots \operatorname{det}\left(\mathcal{A}_{n}\right)\right]^{2^{(n-2) / 2}} .
$$

It follows from Eq. (5) that if one of $\operatorname{det} M\left(\left|\psi^{\prime}\right\rangle\right)$ and $\operatorname{det} M(|\psi\rangle)$ vanishes while the other does not, then the state $\left|\psi^{\prime}\right\rangle$ is not equivalent to $|\psi\rangle$ under SLOCC. In view of the fact that the determinant of a matrix is nonvanishing if and only if it has full rank, the SLOCC invariance of the rank is stronger than the invariance of the determinant.

\section{SLOCC CLASSIFICATION IN TERMS OF THE RANK}

We define the family $\mathcal{F}_{n, r}$ to be the set of all $n$-qubit states with the same rank $r$. In the sequel, we will omit the subscript $n$ and simply write $\mathcal{F}_{r}$, whenever the number of qubits is clear from the context. Thus, there exist $2^{[n / 2]}$ different SLOCC families for any $n$ qubits. Clearly, if two states are SLOCC equivalent then they belong to the same family. However, the converse does not hold: two states belonging to the same family may be inequivalent under SLOCC. It is further noted that when $n \geq 4$, at least one family contains an infinite number of SLOCC classes.

For any $n$ qubits, the following hold: (i) Family $\mathcal{F}_{1}$ contains all the full separable states. (ii) Family $\mathcal{F}_{1}$ contains no genuine entangled states. (iii) Family $\mathcal{F}_{1}$ contains finite SLOCC classes. (iv) Family $\mathcal{F}_{2}$ contains the $n$-qubit $|\mathrm{GHZ}\rangle$ state. (v) Family $\mathcal{F}_{2+r}$ contains the following state:

$$
\frac{1}{\sqrt{r+2}}\left(|0\rangle-\left|2^{n}-1\right\rangle+\sum_{k=1}^{r}\left|k\left(2^{[(n+1) / 2]}+1\right)\right\rangle\right),
$$

where $1 \leq r \leq 2^{[n / 2]}-2$.

Now we turn to the $n$-qubit symmetric Dicke states $|\ell, n\rangle$ with $\ell$ excitations [22]:

$$
|\ell, n\rangle=\left(\begin{array}{c}
n \\
\ell
\end{array}\right)^{-1 / 2} \sum_{k} P_{k}\left|1_{1}, 1_{2}, \ldots, 1_{\ell}, 0_{\ell+1}, \ldots, 0_{n}\right\rangle,
$$

where $\ell$ ranges from 1 to $n-1$ and $\left\{P_{k}\right\}$ is the set of all distinct permutations of the spins. These states have been featured in theoretical studies 23, 24] and implemented experimentally [25, 26]. The Dicke state $|1, n\rangle$ is just the $n$-qubit $|W\rangle$ state and $|\ell, n\rangle$ is equivalent to $|n-\ell, n\rangle$ under SLOCC.

As has been previously noted, all symmetric Dicke states $|\ell, n\rangle$ with $\ell=1, \ldots,[n / 2]$ are SLOCC inequivalent [14, 15]. These states, as demonstrated below, can also be distinguished by the rank of their coefficient matrices which depends only on the number of excitations and is independent of the number of qubits. Since $\operatorname{rank}(|\ell, n\rangle)=\operatorname{rank}(|n-\ell, n\rangle)$, we only need to compute $\operatorname{rank}(|\ell, n\rangle)$ with $1 \leq \ell \leq[n / 2]$. This can be done as follows. We first construct the coefficient matrix $M\left(\left|\psi^{\prime}\right\rangle\right)$ of state $\left|\psi^{\prime}\right\rangle$ in the same manner as discussed above. We may write $\left|\psi^{\prime}\right\rangle$ in terms of an orthogonal basis as $\left|\psi^{\prime}\right\rangle=\sum a_{i_{1} i_{2} \ldots i_{n}}\left|i_{1} i_{2} \ldots i_{n}\right\rangle$, where $i_{1} i_{2} \ldots i_{n}$ is the $n$ bit binary form of the index $i$. Inspection of the structure of the matrix $M\left(\left|\psi^{\prime}\right\rangle\right)$ reveals that the coefficient $a_{i_{1} \ldots i_{[n / 2]} i_{[n / 2]+1} \ldots i_{n}}$ is the entry in the $\left(i_{1} \ldots i_{[n / 2]}\right)$ th row and $\left(i_{[n / 2]+1} \ldots i_{n}\right)$ th column of the matrix. Here, the $n$ bits are split into two halves, referred to as the row bits and column bits, respectively: bits 1 to $[n / 2]$ are used to specify the row number, and bits $[n / 2]+1$ to $n$ are 
used to specify the column number. In view of Eq. (7), the nonzero entries of the coefficient matrix $M(|\ell, n\rangle)$ are those whose $n$-bit binary forms have $\ell$ bits equal to 1 and the rest of the bits equal to 0 . We further observe that the rows of $M(|\ell, n\rangle)$ with no more than $\ell$ row bits equal to 1 are nonzero rows, while the remaining rows are identically zero. Consider the rows with $j(0 \leq j \leq \ell)$ row bits equal to 1 . Clearly, there are $\left(\begin{array}{c}n / 2 \\ j\end{array}\right)$ such rows of $M(|\ell, n\rangle)$ that are identical. Letting $j$ vary from 0 to $\ell$ gives a total of $\ell+1$ different rows. It can be verified that these $\ell+1$ rows are independent. This yields $\operatorname{rank}(|\ell, n\rangle)=\ell+1$.

Accordingly, for any $n$ qubits, all the Dicke states $|\ell, n\rangle$ with $\ell=1, \ldots,[n / 2]$, are inequivalent under SLOCC, since they differ in their ranks. Further, it can readily be seen that the Dicke state $|\ell, n\rangle$ with $\ell=1, \ldots,[n / 2]$ belongs to the family $\mathcal{F}_{\ell+1}$. This gives rise to a complete SLOCC classification of all the symmetric Dicke states.

Remark 2. It follows from the discussion above that both the states $|W\rangle$ and $|\mathrm{GHZ}\rangle$ have the same rank, thereby revealing that the two states have a similar algebraic structure. It is further noted that both the states $|W\rangle$ and $|\mathrm{GHZ}\rangle$ admit a similar Frobenius algebra structure [27].

\section{RANKS OF COEFFICIENT MATRICES UNDER PERMUTATIONS OF QUBITS}

In [17, 18], we presented a systematic way to find all the possible coefficient matrices for even $n$-qubit states such that the determinants of these coefficient matrices are invariant under SLOCC. Here we extend this construction to general $n$ qubits. Observe that to write a $2^{[n / 2]} \times 2^{[(n+1) / 2]}$ matrix into binary index form, we need $[n / 2]$ row bits and $[(n+1) / 2]$ column bits. In the binary form of the coefficient matrix given in Eqs. (11) and (2), bits 1 to $[n / 2]$ are the row bits, and bits $[n / 2]+1$ to $n$ are the column bits. Alternatively, we may choose any $[n / 2]$ bits as the row bits and the remaining $[(n+1) / 2]$ bits as the column bits. This amounts to $\left(\frac{1}{2}\right)^{n+1} \bmod 2\left(\begin{array}{c}n \\ {[n / 2]}\end{array}\right)$ different coefficient matrices, ignoring those that end up exchanging rows or columns. The factor of $1 / 2$ for even $n$ arises because exchanging the row and column bits is equivalent to transposing the matrix. It turns out that the ranks of these coefficient matrices are all invariant under SLOCC. To see this, we will resort to permutations of qubits. Let $\sigma$ be a permutation of qubits given by [18]

$$
\sigma=\left(q_{1}, t_{1}\right)\left(q_{2}, t_{2}\right) \ldots\left(q_{k}, t_{k}\right),
$$

where $\left(q_{i}, t_{i}\right)$ is the transposition of a pair of qubits $q_{i}$ and $t_{i}$ with $q_{i}$ being a row bit and $t_{i}$ a column bit. Exhausting all possible values of $q_{1}, \ldots, q_{k}$ and $t_{1}, \ldots, t_{k}$ such that $1 \leq q_{1}<q_{2}<\ldots<q_{k}<[(n+1) / 2]$, $[n / 2]<t_{1}<t_{2}<\ldots<t_{k} \leq n$, and letting $k$ vary from 0 to $[(n-1) / 2]$ (we define $\sigma=I$ for $k=0$ ), yields

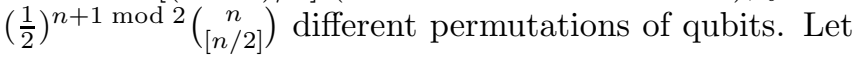
$M^{\sigma}\left(\left|\psi^{\prime}\right\rangle\right)$ denote the coefficient matrix of the state $\left|\psi^{\prime}\right\rangle$ under permutation $\sigma$, and let $\operatorname{rank}^{\sigma}\left(\left|\psi^{\prime}\right\rangle\right)$ denote its rank. We may refer to $\operatorname{rank}^{\sigma}\left(\left|\psi^{\prime}\right\rangle\right)$ as the rank of the state $\left|\psi^{\prime}\right\rangle$ under permutation $\sigma$. Simply taking the permutation $\sigma$ to both sides of Eq. (4) yields the following SLOCC matrix equation:

$$
\begin{array}{r}
M^{\sigma}\left(\left|\psi^{\prime}\right\rangle\right)=\left(\mathcal{A}_{\sigma(1)} \otimes \ldots \otimes \mathcal{A}_{\sigma([n / 2])}\right) M^{\sigma}(|\psi\rangle) \\
\left(\mathcal{A}_{\sigma([n / 2]+1)} \otimes \ldots \otimes \mathcal{A}_{\sigma(n)}\right)^{T} .
\end{array}
$$

It follows immediately from Eq. (9) that two SLOCC equivalent states have the same rank with respect to the permutation $\sigma$. That is, the rank with respect to the permutation $\sigma$ is invariant under SLOCC. Conversely, if two states differ in their ranks with respect to the permutation $\sigma$, then they belong necessarily to different SLOCC classes. We define the family $\mathcal{F}_{r}^{\sigma}$ to be the set of all $n$ qubit states with the same rank $r$ with respect to the permutation $\sigma$, where $r$ ranges from 1 to $2^{[n / 2]}$ and we have omitted a subscript $n$. Suppose $\sigma_{1}, \sigma_{2}, \ldots, \sigma_{m}$ with $m \leq\left(\frac{1}{2}\right)^{n+1 \bmod 2}\left(\begin{array}{c}n \\ {[n / 2]}\end{array}\right)$ is a sequence of permutations of the form given in Eq. (8). In terms of the rank of $M^{\sigma_{1}}$, the $n$-qubit states are divided into $2^{[n / 2]}$ families: $\mathcal{F}_{r_{1}}^{\sigma_{1}}$. Then, in terms of the rank of $M^{\sigma_{2}}$, each family $\mathcal{F}_{r_{1}}^{\sigma_{1}}$ can be further divided into $2^{[n / 2]}$ subfamilies: $\mathcal{F}_{r_{1}, r_{2}}^{\sigma_{1} \sigma_{2}}=\mathcal{F}_{r_{1}}^{\sigma_{1}} \cap \mathcal{F}_{r_{2}}^{\sigma_{2}}$. Here, each subfamily $\mathcal{F}_{r_{1}, r_{2}}^{\sigma_{1} \sigma_{2}}$ is the intersection of the families $\mathcal{F}_{r_{1}}^{\sigma_{1}}$ and $\mathcal{F}_{r_{2}}^{\sigma_{2}}$. Assume that in terms of the ranks of $M^{\sigma_{1}}, M^{\sigma_{2}}, \ldots, M^{\sigma_{m-1}}$, the $n$-qubit states are divided into $2^{(m-1)[n / 2]}$ families: $\mathcal{F}_{r_{1}, r_{2}, \ldots, r_{m-1}}^{\sigma_{1} \sigma_{2} \ldots \sigma_{m-1}}=\mathcal{F}_{r_{1}}^{\sigma_{1}} \cap \ldots \cap \mathcal{F}_{r_{m-1}}^{\sigma_{m-1}}$. Then, in terms of the rank of $M^{\sigma_{m}}$, each family $\mathcal{F}_{r_{1}, r_{2}, \ldots, r_{m-1}}^{\sigma_{1} \sigma_{2} \ldots \sigma_{m-1}}$ can be further divided into $2^{[n / 2]}$ subfamilies: $\mathcal{F}_{r_{1}, r_{2}, \ldots, r_{m}}^{\sigma_{1} \sigma_{2} \ldots \sigma_{m}}=$ $\mathcal{F}_{r_{1}, r_{2}, \ldots, r_{m-1}}^{\sigma_{1} \sigma_{2} \ldots \sigma_{m-1}} \cap \mathcal{F}_{r_{m}}^{\sigma_{m}}=\mathcal{F}_{r_{1}}^{\sigma_{1}} \cap \ldots \cap \mathcal{F}_{r_{m}}^{\sigma_{m}}$. This gives a total of $2^{m[n / 2]}$ different SLOCC families.

We exemplify with the family $L_{a_{2} b_{2}}=a(|0000\rangle+$ $|1111\rangle)+b(|0101\rangle+|1010\rangle)+|0011\rangle+|0110\rangle$ for four qubits presented by Verstraete et al. [3]. As shown in Table I, the family $L_{a_{2} b_{2}}$ is further divided into four subfamilies (all other subfamilies are empty) with respect to permutations $\sigma_{1}=I$ and $\sigma_{2}=(1,4): \mathcal{F}_{2,1}^{\sigma_{1} \sigma_{2}}, \mathcal{F}_{3,3}^{\sigma_{1} \sigma_{2}}$, and $\mathcal{F}_{4,2}^{\sigma_{1} \sigma_{2}}$ contain only a single SLOCC class, while $\mathcal{F}_{4,3}^{\sigma_{1} \sigma_{2}}$ contains an infinite number of SLOCC classes. In a similar fashion, we can further divide other families presented by Verstraete et al. [3] into subfamilies.

TABLE I. SLOCC classification of $L_{a_{2} b_{2}}$

\begin{tabular}{cccc}
\hline \hline $\mathcal{F}_{1}^{\sigma_{1}}$ & $\mathcal{F}_{2}^{\sigma_{1}}$ & $\mathcal{F}_{3}^{\sigma_{1}}$ & $\mathcal{F}_{4}^{\sigma_{1}}$ \\
$\emptyset$ & $a=b=0$ & $a b=0 \& a \neq b$ & $a b \neq 0$ \\
$\mathcal{F}_{1}^{\sigma_{2}}$ & $\mathcal{F}_{2}^{\sigma_{2}}$ & $\mathcal{F}_{3}^{\sigma_{2}}$ & $\mathcal{F}_{4}^{\sigma_{2}}$ \\
$a=b=0$ & $a= \pm b \& a \neq 0$ & $a \neq \pm \neq$ & $\emptyset$ \\
$\mathcal{F}_{2,1}^{\sigma_{1} \sigma_{2}}$ & $\mathcal{F}_{3,3}^{\sigma_{1} \sigma_{2}}$ & $\mathcal{F}_{4,2}^{\sigma_{1} \sigma_{2}}$ & $\mathcal{F}_{4,3}^{\sigma_{1} \sigma_{2}}$ \\
$a=b=0$ & $a b=0 \& a \neq b$ & $a= \pm b \& a \neq 0 \quad a b \neq 0 \& a \neq \pm b$ \\
\hline \hline
\end{tabular}


Consider the family $\operatorname{span}\left\{0_{k} \Psi, 0_{k} \Psi\right\}=|0000\rangle+$ $|1100\rangle+\alpha|0011\rangle+\beta|1111\rangle$ for four qubits presented by Lamata et al. [5]. As shown in Table [I] the family $\left\{0_{k} \Psi, 0_{k} \Psi\right\}$ is further divided into four subfamilies (all other subfamilies are empty) with respect to permutations $\sigma_{1}=I$ and $\sigma_{2}=(1,4): \mathcal{F}_{1,2}^{\sigma_{1} \sigma_{2}}, \mathcal{F}_{1,4}^{\sigma_{1} \sigma_{2}}$, and $\mathcal{F}_{2,3}^{\sigma_{1} \sigma_{2}}$ contain only a single SLOCC class, while $\mathcal{F}_{2,4}^{\sigma_{1} \sigma_{2}}$ contains an infinite number of SLOCC classes. In a similar way, other families presented by Lamata et al. [5] can also be further divided into subfamilies.

TABLE II. SLOCC classification of $\operatorname{span}\left\{0_{k} \Psi, 0_{k} \Psi\right\}$

\begin{tabular}{cccc}
\hline \hline $\mathcal{F}_{1}^{\sigma_{1}}$ & $\mathcal{F}_{2}^{\sigma_{1}}$ & $\mathcal{F}_{3}^{\sigma_{1}}$ & $\mathcal{F}_{4}^{\sigma_{1}}$ \\
$\alpha=\beta$ & $\alpha \neq \beta$ & $\emptyset$ & $\emptyset$ \\
$\mathcal{F}_{1}^{\sigma_{2}}$ & $\mathcal{F}_{2}^{\sigma_{2}}$ & $\mathcal{F}_{3}^{\sigma_{2}}$ & $\mathcal{F}_{4}^{\sigma_{2}}$ \\
$\emptyset$ & $\alpha=\beta=0$ & $\alpha \beta=0 \& \alpha \neq \beta$ & $\alpha \beta \neq 0$ \\
$\mathcal{F}_{1,2}^{\sigma_{1} \sigma_{2}}$ & $\mathcal{F}_{1,4}^{\sigma_{1} \sigma_{2}}$ & $\mathcal{F}_{2,3}^{\sigma_{1} \sigma_{2}}$ & $\mathcal{F}_{2,4}^{\sigma_{1} \sigma_{2}}$ \\
$\alpha=\beta=0$ & $\alpha=\beta \neq 0$ & $\alpha \beta=0 \& \alpha \neq \beta$ & $\alpha \beta \neq 0 \& \alpha \neq \beta$ \\
\hline \hline
\end{tabular}

By using the filters, it has been shown that four fivequbit states $\left|\Psi_{2}\right\rangle,\left|\Psi_{4}\right\rangle,\left|\Psi_{5}\right\rangle$, and $\left|\Psi_{6}\right\rangle$ are in different orbits [28]. Letting $\sigma_{1}=I, \sigma_{2}=(1,5)$, and $\sigma_{3}=(1,3)$, it can be shown that the above four states belong to the families $\mathcal{F}_{2,2,2}^{\sigma_{1} \sigma_{2} \sigma_{3}}, \mathcal{F}_{3,3,3}^{\sigma_{1} \sigma_{2} \sigma_{3}}, \mathcal{F}_{2,4,2}^{\sigma_{1} \sigma_{2} \sigma_{3}}$ and $\mathcal{F}_{2,4,4}^{\sigma_{1} \sigma_{2} \sigma_{3}}$, respectively. Therefore, these five-qubit states can also be distinguished by ranks. Furthermore, it has been shown that five six-qubit states $\left|\Xi_{2}\right\rangle,\left|\Xi_{4}\right\rangle,\left|\Xi_{5}\right\rangle,\left|\Xi_{6}\right\rangle$, and $\left|\Xi_{7}\right\rangle$ are distinguished by the six-qubit filters [28]. Letting $\sigma_{1}=I, \sigma_{2}=(1,4)$, and $\sigma_{3}=(1,5)$, it can be shown that the above five states belong to the families $\mathcal{F}_{2,2,2}^{\sigma_{1} \sigma_{2} \sigma_{3}}$, $\mathcal{F}_{2,2,4}^{\sigma_{1} \sigma_{2} \sigma_{3}}, \mathcal{F}_{2,4,4}^{\sigma_{1} \sigma_{2} \sigma_{3}}, \mathcal{F}_{3,4,4}^{\sigma_{1} \sigma_{2} \sigma_{3}}$, and $\mathcal{F}_{3,3,3}^{\sigma_{1} \sigma_{2} \sigma_{3}}$, respectively. Therefore, these six-qubit states can also be distinguished by ranks.

\section{DISCUSSION AND SUMMARY}

Chterental et al. (see Remark 3.5 in [4]) stated that the family $L_{a b_{3}}$ is equivalent to a subfamily of $L_{a b c_{2}}$ obtained by setting $a=c$, where $L_{a b_{3}}$ and $L_{a b c_{2}}$ are given by [3]

$$
\begin{aligned}
L_{a b_{3}}= & a(|0000\rangle+|1111\rangle)+\frac{a+b}{2}(|0101\rangle+|1010\rangle) \\
& +\frac{a-b}{2}(|0110\rangle+|1001\rangle)+\frac{i}{\sqrt{2}}(|0001\rangle+|0010\rangle \\
& +|0111\rangle+|1011\rangle), \\
L_{a b c_{2}}= & \frac{a+b}{2}(|0000\rangle+|1111\rangle)+\frac{a-b}{2}(|0011\rangle+|1100\rangle) \\
& +c(|0101\rangle+|1010\rangle)+|0110\rangle .
\end{aligned}
$$

In terms of the rank, the families $L_{a b_{3}}$ and $L_{a b c_{2}}$ with $a=c$ are both divided into four subfamilies, see Table III. As can be seen, the subfamily $\mathcal{F}_{2}$ of $L_{a b_{3}}$ is a single class with representative $\frac{i}{\sqrt{2}}(|0001\rangle+|0010\rangle+|0111\rangle+|1011\rangle)$, whereas the subfamily $\mathcal{F}_{2}$ of $L_{a b c_{2}}(a=c)$ is a single class with representative $\frac{b}{2}(|0000\rangle+|1111\rangle-|0011\rangle-|1100\rangle)+$ $|0110\rangle$. In light of Theorem 1 in [20], the two representative states are not equivalent to each other. This reveals that $L_{a b_{3}}$ is not equivalent to a subfamily of $L_{a b c_{2}}$ obtained by setting $a=c$.

TABLE III. SLOCC classifications of $L_{a b_{3}}$ and $L_{a b c_{2}}$

\begin{tabular}{ccccc}
\hline \hline$L_{a b 3}$ & $\mathcal{F}_{1}$ & $\mathcal{F}_{2}$ & $\mathcal{F}_{3}$ & $\mathcal{F}_{4}$ \\
& $\emptyset$ & $a=b=0$ & $a b=0 \& a \neq b$ & $a b \neq 0$ \\
$L_{a b c_{2}}$ & $\mathcal{F}_{1}$ & $\mathcal{F}_{2}$ & $\mathcal{F}_{3}$ & $\mathcal{F}_{4}$ \\
$(a=c)$ & $a=b=0$ & $a=0 \& b \neq 0$ & $a \neq 0 \& b=0$ & $a b \neq 0$ \\
\hline \hline
\end{tabular}

To determine if a four-qubit state belongs to a family according to the criteria given by Verstraete et al. 3] and Lamata et al. [5], one needs to check if the state is equivalent to the representative state of that family. For the classification scheme proposed in this Letter, to determine if an $n$-qubit state belongs to a family, one needs only to calculate the rank of the coefficient matrix of the state.

In summary, we have studied SLOCC classification for general $n$-qubit states via the invariance of the rank of the coefficient matrix and given several examples for $n$ up to six. We have also characterized full separable states and genuinely entangled states in terms of the rank. We expect that the proposed entanglement classification for general $n$-qubit states may find further experimental consequences.

This work was supported by NSFC (Grant No. 10875061) and Tsinghua National Laboratory for Information Science and Technology.

[1] M.A. Nielsen and I.L. Chuang, Quantum Computation and Quantum Information (Cambridge Univ. Press, Cambridge, 2000).

[2] W. Dür, G. Vidal, and J.I. Cirac, Phys. Rev. A 62, 062314 (2000).

[3] F. Verstraete, J. Dehaene, B. De Moor, and H. Verschelde, Phys. Rev. A 65, 052112 (2002).

[4] O. Chterental and D.Z. Djoković, in Linear Algebra Research Advances, edited by G.D. Ling (Nova Science Publishers, Inc., Hauppauge, NY, 2007), Chap. 4, 133.

[5] L. Lamata, J. León, D. Salgado, and E. Solano, Phys. Rev. A 75, 022318 (2007).

[6] L. Borsten, D. Dahanayake, M.J. Duff, A. Marrani, and W. Rubens, Phys. Rev. Lett. 105, 100507 (2010).

[7] Y. Cao and A.M. Wang, Eur. Phys. J. D 44, 159 (2007).

[8] D. Li, X. Li, H. Huang, and X. Li, Phys. Rev. A 76, 052311 (2007);

[9] D. Li, X. Li, H. Huang, and X. Li, Quantum Inf. Comput. 9, 0778 (2009).

[10] R.V. Buniy and T.W. Kephart, arXiv:1012.2630.

[11] O. Viehmann, C. Eltschka, and J. Siewert, Phys. Rev. A 83, 052330 (2011).

[12] X. Zha and G. Ma, Chin. Phys. Lett. 28, 020301 (2011). 
[13] L. Chen and Y.X. Chen, Phys. Rev. A 74, 062310 (2006).

[14] D. Li, X. Li, H. Huang, and X. Li, Europhys. Lett. 87, 20006 (2009).

[15] T. Bastin, S. Krins, P. Mathonet, M. Godefroid, L. Lamata, and E. Solano, Phys. Rev. Lett. 103, 070503 (2009).

[16] M. Aulbach, arXiv:1103.0271.

[17] X. Li and D. Li, J. Phys. A: Math. Theor. 44, 155304 (2011).

[18] X. Li and D. Li, J. Phys. A: Math. Theor. 45, 075308 (2012).

[19] X. Li and D. Li, Quantum Inf. Comput. 11, 0695 (2011).

[20] D. Li, X. Li, H. Huang, and X. Li, Phys. Rev. A 76, 032304 (2007).

[21] P. Ribeiro and R. Mosseri, Phys. Rev. Lett. 106, 180502 (2011).
[22] J.K. Stockton, J.M. Geremia, A.C. Doherty, and H. Mabuchi, Phys. Rev. A 67, 022112 (2003).

[23] G. Tóth, J. Opt. Soc. Am. B 24, 275 (2007).

[24] M. Huber, P. Erker, H. Schimpf, A. Gabriel, and B. Hiesmayr, Phys. Rev. A 83, 040301(R) (2011).

[25] R. Prevedel, G. Cronenberg, M.S. Tame, M. Paternostro, P. Walther, M.S. Kim, and A. Zeilinger, Phys. Rev. Lett. 103, 020503 (2009).

[26] W. Wieczorek, R. Krischek, N. Kiesel, P. Michelberger, G. Tóth, and H. Weinfurter, Phys. Rev. Lett. 103, 020504 (2009).

[27] B. Coecke and A. Kissinger, arXiv:1002.2540.

[28] A. Osterloh and J. Siewert, Int. J. Quant. Inf. 4, 531 (2006). 